\title{
STUDIES ON CRYSTAL SEMI-PERMEABLE MEMBRANE IV. CONFIRMATION ON FORMATION CONDITION OF PFEFFER'S SEMI-PERMEABLE MEMBRANE BY GLASS U-FORMED TUBE
}

\author{
KIYOSHI TERAZAWA \\ Biochemical Department, Medical School, Tokyo Medico-Dental \\ University, Tokyo
}

(Received: October 21st, 1953)

INTRODUCTION

The authors built up a concept of crystal semi-permeable membrane, stating in the $1 \mathrm{st}^{1)}$ and $2 \mathrm{nd}^{2}$ ) reports that a phenomenon of a semi-permeable membrane formation of silicate reported by Miyamoto and Takikawa ${ }^{3)}$ is not peculiar in silicate but it is able to be caused by all which form water-insoluble salts at the Pringsheim's equilibrium point. In the 3rd report4), moreover, a copper ferrocyanide semi-permeable membrane, a representative of the membrane made of inorganic substances, in the Pfeffer's unglazed pottery cylinder, was reported and confirmed to be a kind of the crystal semi-permeable membrane called by the authors.

In a model experiment on the Pfeffer's semi-permeable membrane in this report, glass U-formed tube was used instead of unglazed pottery cylinder, to confirm, furthermore, the already stated fact by an observation in practice on a proceeding state of precipitate which was not able to be directly examined.

\section{METHoD}

The experiment in this report was carried out by the following method: Agar sol was poured into the glass U-formed tube in a degree to fill its flexure. After the sol was changed into gel, copper sulfate solution with a certain constant concentration was poured into one side of the U-formed tube and potassium ferrocyanide solutions gradually changed in concentration into the other side. At that time, each solution infiltrated into agar gel from the both sides and a precipitate of copper ferrocyanide was formed inside the gel. Observation was made on the concentration relation of both solutions in which the precipitate formed a semi-permeable membrane, and on the proceeding state of the precipitate. Further study was taken on a degree of diffusion of congo red solution to examine a permeability of the precipitate layer or membrane. This experiment used the glass U-formed tube full of agar instead of an unglazed pottery cylinder and therefore a detailed examination was made on the proceeding state of the precipitate, different from the case of the pottery cylinder in the 3rd report. 
Stating the experimental order in detail, in the first place the glass Uformed tube, of about $8 \mathrm{~cm}$. high, about $0.8 \mathrm{~cm}$. in internal diameter, about $3 \mathrm{~cm}$. in between both center of it was in advance water-washed and dried up. Two \% agar sol was poured into the flexure part of each tube and was put untouchedly for hours at room temperature to be changed into a complete gel. Nextly each 2 cc. of $1 \%$ copper sulfate solution into one side of the U-tube and potassium ferrocyanide solution into the other side was simultaneously poured (Fig. 1). While copper sulfate solution was kept constantly $1 \%$ in concentration, 11 kinds in concentration of potassium ferocyanide, 0.1, 0.25, 0.5, 0.75, 1.0, 1.25, 1.5, 1.75, 2.0, 2.25 and $2.5 \%$, were taken just like in the case of the 3rd report. Those tubes were kept perpendicularly and untouchedly at room temperature. In five days when the precipitate proceeded considerably, both solutions were got out, and the tubes were quietly water-washed at several time. Thereafter, the tubes were filled up by water and kept untouchedly for about 12 hours to diffuse and exclude non-reacted electrolyte as much as possible. $0.5 \%$ congo red solution was poured into the side of potassium ferrocyanide and water into the other side at the same height. Those tubes were kept untouchedly at room temperature for about one month. Thus, an observation was made on the permeation of the pigment throught the precipitate layer formed in agar.

\section{RESULT}

\section{A. The State of the Precipitate}

1. In the case of thin potassium ferrocyanide solution: In a small extent of potassium ferrocyanide solution in cencentration for copper sulfate solution in the other side, that is, in under $1 \%$ concentration, both electrolytes diffused from the both sides of U-tube, came into collision about at the center of the gel or a little near the potassium ferrocyanide side, where a thin membrane of copper ferrocyanide is formed. With its membrane as a starting surface, the precipitate proceeds towards the side of comparatively thin potassium ferrocyanide. In the case, a thin membraneous precipitate by copper ferrocyanide caused by a collision of the both solution is far thinner in color than in the followingly stated case of thick potassium ferrocyanide solution. As the precipitate proceeds, the color gradually increases (Fig. 2, a, Photo).

2. In the case of thick potassium ferrocyanide solution: On the contrary, in a large extent in concentration of potassium ferrocyanide solution, that is, in above $1.75 \%$, the both solutions come into collision a little near the side of copper sulfate in the gel layer to form a thin membrans of copper ferrocyanide. With it as a starting surface, the precipitate proceeds towards the side of copper sulfate. In the previous case of the thin potassium ferocyanide, the precipitate layer becomes dense in color as it proceeds, but in this case, a dense precipitate 
is formed in the first collision and the layer caused thereafter keeps constant in concentration for the way of its proceeding (Fig. 2, c, Photo).

3. Equilibrium Point: In its intermediate concentration, that is, in $\mathbf{1 . 2 5}$ and $1.5 \%$ of potassium ferrocyanide, the one and copper sulfate diffused from the both sides comes into collision, a little inclined to the side of copper sulfate in the gel layer to form a thin membrane as above mentioned, but it proceeds towards neither side and as the time passes a seemingly closed thin membrane only gets formed (Fig. 2, b, Photo).

\section{B. Proceeding State of Precipitate}

Two different kinds of phenomena were observed in the proceeding state of precipitate. The first phenomenon is as observed in the case of the thin potassium ferrocyanide solution as previously mentioned. At the collided part of the both solutions in their diffusion into the agar, a thin membraneous precipitate of copper ferrocyanide was formed, which is thin in color, compared to the case of thick potassium ferrocyanide. This precipitate concentration increases gradually density as it proceeds. In other words, the thickest precipitate is always formed in the front to the proceeding way of the layer. This phenomenon will be explained in the followingly mentioned mechanism: After the first precipitate formation at the collided part, highly concentrated copper sulfate invaded into the other side of low potassium ferrocyanide through its membrane and to its layer gradually proceeded and developed towards the ferrocyanide side. It is quite noteworthy that the longer the layer is, the lower the diffusion of $\mathrm{Cu}^{++}$anion is in speed, when the precipitate permeats through copper sulfate, because corpuscles of insoluble copper ferrocyanide in the precipitate layer are crystallized and the corpuscles have negative ion for the agar. Ferrocyanide ion $\mathrm{Fe}(\mathrm{CN})_{6}{ }^{----}$to diffuse to the contrary way formed a precipitate just in front of the precipitate layer with low $\mathrm{Cu}++$ is speed which permeated just though the layer.

The first phenomenon will be understood by such an explanation that the lower $\mathrm{Cu}^{++}$gets in diffusion speed, the larger insoluble copper ferrocyanide produced in a unit distance may become in concentration, because ferrocyanide in constant speed comes into collision with low-speeded $\mathrm{Cu}++$

On the contrary, the second phenomenon is observed in the case of thick potassium ferrocyanide solution as previously mentioned. In this case, such a phenomenon was not observed that the layer's front formed a still thicker precipitate for the proceeding way.

Because ferrocyanide ion permeats possibly through interstices among corpuscles of copper ferrocyanide crystallized in the layer with negative charge, the problem of charges is not concerned with the phenomenon. In the case of thick potassium ferrocyanide, a mechanical action to disturb its permeation have a great influence, while there is no influence of charge, because a thick 
precipitate is formed from the start. Therefore, the late formation of the whole precipitate layer appears rather than change in concentration of the layer because a decrease of permeated quantity is caused strongly contrary to a decrease in diffusion speed as in the previous case, it is thought.

\section{SUMMARY AND DISCUSSION}

The study in the 3rd report on a series of these experiments followed the experiment on the semi-permeable membrane of copper ferrocyanide in the Pfeffer's unglazed pottery cylinder changing the internal and external electrolytes in concentration. As a result, it was confirmed that the semi-permeable membrane was formed only at a balanced concentration of internal and external electrolytes, and there may be an equivalent relation in its equilibrial concentration. The same experiment was carried out by the use of the U-formed tube in this report in which a dynamic observation was made in agar gel layer on the same phenomenon as in the cutting surface of unglazed pottery cylinder's wall after its reaction. It was known that the appearance of diffusion and the proceeding way of the precipitate layer was subject to a very regular rule. Various phenomena were easily explained by the thought that non-dissociated and insoluble copper ferocyanide corpuscles crystallized in the precipitate layer had a negative charge in these surface according to a general colloid law. While it is different from the main aim of this study, of course, it is thought that an interesting problem was proposed to clear this kind of phenomenon up in future in the fact that one of the most important condition was discovered, in the case of which one electrolyte permeated through the precipitate layer by concentration difference between both electrolytes after an insoluble precipitate was formed coming into collision of electrolytes in colloid in general.

Judging from these matters, such a very thick precipitate layer is formed only in an equivalent relation of concentrations between the both electrolytes when two electrolytes which form an insoluble salt precipitate come into collision in their border surface, that even pigment can not permeate, while in the case of unbalanced concentration, an impermeable membrane will not be formed even in the case of much precipitate formation, it was clearly understood. It may be impossible to recognize that there is no mechanical distrubance action for solution to permeate into the thick side for much precipitate and ther is no action to decrease the speed of passing ion influenced by surface charge of non-dissociated and insoluble corpuscles crystallized in the layer. Those actions, however, only decrease its permeability and cannot disturb completely, it was understood. When the layer is formed in a much greater deal, it seems that there happens to be a complete disturbanced for electrolytes permeation. In this case, however, it is still more considerably confirmed that the permeation disturbance action is quite different in its mechanism from the nature of the so-called crystal semi-permeable membrane by the authors formed at the so- 
called "Pringsheim's equilibrium point". It is a matter of course that the nature and mechanism of the crystal semi-permeable membrane are greatly dependent upon its study in future.

\section{CONCLUSION}

1. When the Pfeffer's experiment of copper ferrocyanide semi-permeable membrane in unglazed pottery cylinder was carried out in agar instead, a semipermeable membrane was formed at the Pringsheim's equilibrium point similar with the case of unglazed pottery cylinder.

2. A structure of the so-called crystal semi-permeable membrane by the authors at the Pringsheim's equilibrium point is quite different in its action mechanism from a mechanical disturbance action of permeation indicated by much quantity of thick precipitate layer formed at the time when both solutions, each very thick and equivalently unblanced, some into contact in the border surface.

\section{BIBLIOGRAPHY}

1. K. Terazawa: Ochanomizu Med. J. 1. no. 1 (1952)

2. K. Terazawa: Ochanomizu Med. J. in press

3. Miyamoto and Takikawa: Igaku to Seibutsu Gaku (Medicine and Biology, in Japanese), p. 351 (1942)

4. K. Terazawa: Ochanomizu Med. J., in press 

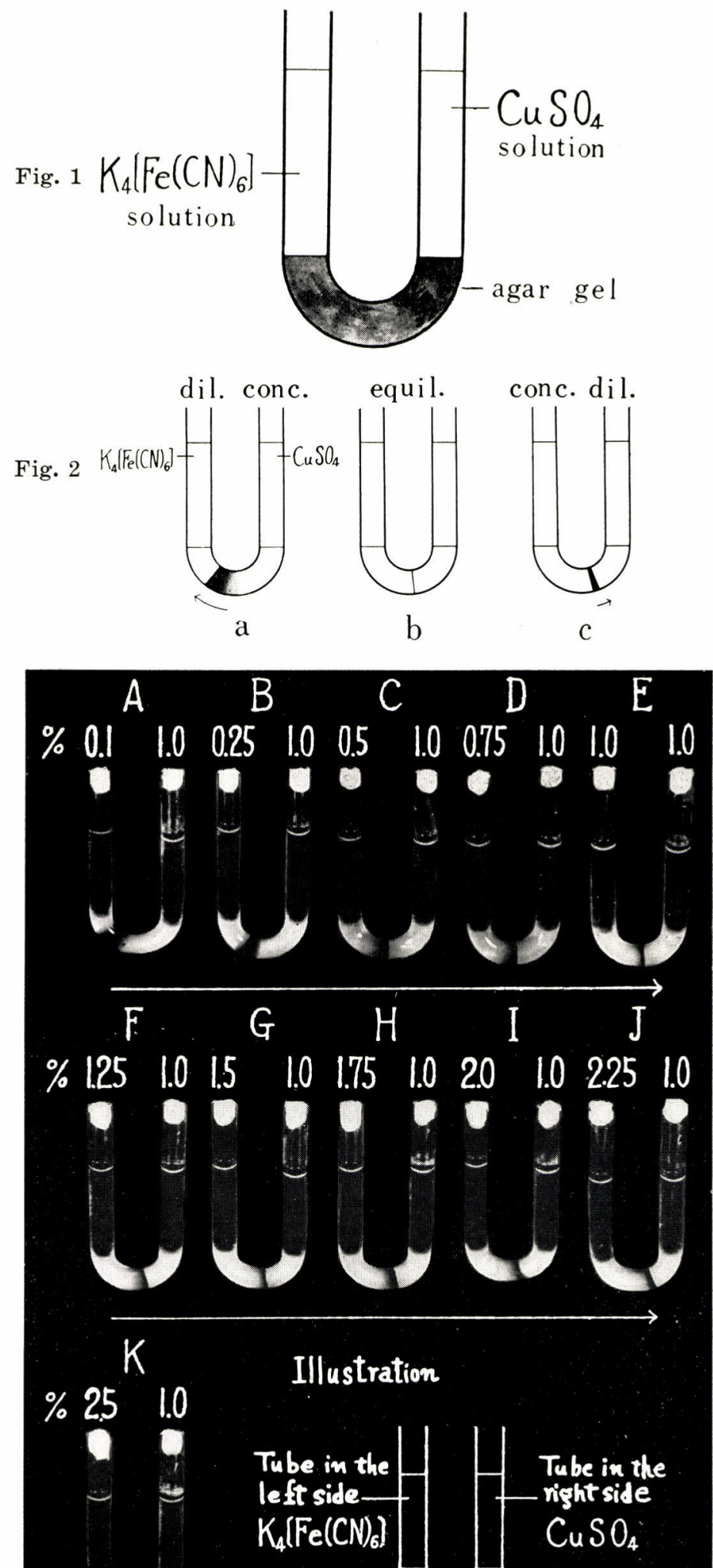

Tube in the right side $\mathrm{CuSO}_{4}$ 